\title{
Technical Aspects of Intensive Care Unit Management: A Single-Center Experience at a Tertiary Academic Hospital
}

\author{
Fahad Alsohime (D) ${ }^{1-3, *}$ \\ Mohamad-Hani Temsah (iD) 1,2,4,* \\ Ayman Al-Eyadhy (D) 1,2 \\ Sanaa Ghulman ${ }^{2}$ \\ Haytam Mosleh ${ }^{2}$ \\ Omar Alsohime ${ }^{4,5}$ \\ 'Pediatric Department, College of \\ Medicine, King Saud University, Riyadh, \\ Saudi Arabia; ${ }^{2}$ Pediatric Intensive Care \\ Unit, King Saud University Medical City, \\ Riyadh, Saudi Arabia; ${ }^{3}$ Clinical Skills \& \\ Simulation Center, King Saud University \\ Medical City, Riyadh, Saudi Arabia; \\ ${ }^{4}$ Prince Abdullah Ben Khalid Celiac \\ Disease Research Chair, King Saud \\ University, Riyadh, Saudi Arabia; \\ ${ }^{5}$ Regulation and Registration Support \\ Department, Medical Devices Sector, \\ Saudi Food and Drug Authority, Riyadh, \\ Saudi Arabia
}

*These authors contributed equally to this work

\begin{abstract}
Purpose: Special technical issues associated with the function and maintenance of medical devices arise in intensive care units (ICUs). This study explored the level of comfort of ICU staff in dealing with selected equipment, the factors that are associated with the staff's ease of adaptation to new technologies, and the role of technical support staff.
\end{abstract}

Patients and Methods: This is a single-center cross-sectional questionnaire-based survey that was conducted in February 2018 and targeted nurses working in the ICUs of King Saud University Medical City in Riyadh, Saudi Arabia.

Results: Among the 297 nurses who completed the survey, almost all of the respondents (99.3\%) were aware of the ICU equipment preventive maintenance program. Most of the nurses had received training on how to use infusion pumps (96.2\%), cardiac monitoring systems $(78.0 \%)$, and cardiac defibrillation devices (73.9\%). Sixty nurses $(20.2 \%)$ indicated that at least one super user was available for at least one device. About half of the staff reported one device whose user manual was available. Most nurses reported having no resources regarding updates on medical devices.

Conclusion: Our findings revealed an alarming need to address technical issues related to medical devices used in the ICU and to design a framework for the safe operation of medical devices based on international practices. It is necessary to empower the role of the super user and medical device clinical educator as well as to optimize communication between the national regulatory body of medical devices and healthcare providers, especially those working in acute care areas.

Keywords: medical devices, ICU, biomedical, safety

\section{Introduction}

Intensive care units (ICUs) follow a multidisciplinary care model that ensures patients receive optimal care. Because the care environment in an ICU is complex, and an efficient workflow requires the knowledge and skills of the intensivist staff who work together to improve patient outcomes and safety.

In addition to relying on a multi-specialty approach to care, ICUs depend heavily on the most advanced technology. Medical devices, including ventilators, infusion pumps, and other consumables, provide an unprecedented opportunity to improve patient care and outcomes. ${ }^{1}$ However, ICU staff may face challenges in safely operating, updating, and maintaining these devices. Additionally, there is the challenge of implementing guidelines to detect device misuse and report adverse events associated with the use of these devices to the appropriate authorities. ${ }^{2}$ Thus,
Correspondence: Fahad Alsohime Pediatric Department, College of Medicine, King Saud University, P. O. Box 2925, Riyadh, I I 46I, Saudi Arabia Tel +96650747929 I

Email dr.fahad.alsohime@gmail.com 
technical support teams should employ of user-friendly technologies to optimize patient care by properly managing technical issues related to different devices in ICUs.

In most ICUs, technical procedures are performed by various team members, most commonly by nursing staff. Although nurses' primary role is patient care, they are usually tasked with managing these technical issues. ${ }^{3}$ Surprisingly, little has been described about the role of technical support staff in ICUs, who are supposed to perform technical procedures, while medical and nursing staff provide medical care for critically ill patients. ${ }^{4-6}$

The objective of this work was to assess ICU healthcare workers' (HCWs') level of awareness of their roles and those of technical staff (biomedical engineers and technicians) in maintaining the safe operation of medical devices.

Specifically, this survey was conducted to 1) explore the level of comfort of different ICU staff in dealing with specific equipment in their work environment based on annex 1 of the German medical devices operation ordinance $^{7}$ 2) explore the factors associated with the ease of adaptation to new technologies, including how ICU staff troubleshoot technical issues and how this process can be improved; and 3) determine whether the role of technical support staff is well-recognized and identify their level of involvement in the unit.

\section{Methods}

\section{Study Design and Participants}

This is a follow-up study of a previously conducted singlecenter, cross-sectional questionnaire-based survey conducted in February 2018 investigating the management of medical devices in ICUs. ${ }^{2}$ The study targeted nurses working in ICUs at King Saud University Medical City (KSUMC), Riyadh, Saudi Arabia. The required representative sample collected to ensure a 95\% confidence level and margin of error less than or equal to $5 \%$ was 218 of the $502(43.4 \%)$ nurses working in the critical care units.

The survey (S1 Form) was distributed to all the critical care units of the hospital, including the Surgical, Medical, Pediatric, and Neonatal ICUs. The nurses were invited to complete the survey according to their preference (electronic or paper-based). The questionnaire was drafted by the authors based on a literature review of adverse events related to medical devices. A combination of evidence appraisals and expert opinions was used to design the questionnaire.
The MEDLINE and PubMed electronic databases were searched for articles in the English language. The search terms included "medical," "device," "reporting," "safety," "usability," and "adverse events OR critical care." Potentially relevant articles were reviewed, and their reference lists were screened to identify other relevant articles. Of them four articles were used to design the questionnaire. ${ }^{8-11}$ Then, a multidisciplinary team produced the final version of the questionnaire (Supplementary mate rial: Appendix 1), which was reviewed by experts from the biomedical engineering department, nursing department, and critical care unit. A pilot survey was conducted in our department to assess the clarity of the questionnaire.

\section{Ethics Statement}

Written informed consent was obtained from all participants. Participation was voluntary, and all participants were assured that their confidential information would be protected. The Institutional Review Board of the King Saud University granted ethical approval to conduct this survey. This study was conducted in accordance with the Declaration of Helsinki.

\section{Questionnaire}

The survey tool was a self-administered questionnaire consisting of three sections. The first section included questions on demographic characteristics (age, gender, credentials, discipline, and work experience). The second section included questions that assessed respondents' overall comfort levels in dealing with various medical devices in the ICU. Participants were asked to rate their overall comfort levels in using different medical devices on a Likert-type scale from one (strongly disagree) to five (strongly agree). They were also asked whether they had received formal training in operating the devices that are typically used critical care settings. Additionally, they were asked whether their unit had a designated super user and how they managed issues that arose with any of the devices. The third section of the questionnaire comprised questions asking the participants to name the assigned person for certain procedures related to the medical devices in the ICU, such as equipment assembly, troubleshooting, supply ordering, etc.

Lastly, the participants were requested to explain how they received updates regarding the different medical devices and what they would do if faced with an issue while operating these devices. 


\section{Statistical Analysis}

Frequencies and percentages were used to describe the categorically measured variables and the mean and standard deviations were used to describe the continuous measured variables. The multiple response dichotomies analysis was used to describe the questions measured with multiple selections.

The statistical significance of the HCWs' perceived responsibility for troubleshooting medical devices assigned to different personnel was assessed using the non-parametric chi-square goodness-of-fit test. The test was performed under the assumption that $\mathrm{HCWs}$ would assign equal responsibility to every person as the a posteriori hypothesis (expected assumption) when compared with the observed distribution.

\section{Results}

\section{Demographic Data}

Of the 502 nurses working in the critical care units of the KSUMC, 297 responded to the survey, representing a response rate of $59 \%$. The respondents were from different critical care disciplines, comprising staff from the medical ICU (29.3\%), surgical ICU (21.9\%), pediatric ICU (29.3\%), and neonatal ICU (19.5\%). Most of the nurses had 6-10 years' work experience (26.3\%), followed by those with 3-5 years' and more than 10 years' experience $(24.9 \%$ each) (Table 1$)$.

\section{Periodic Preventative Maintenance (PPM)}

Most of the participants (99.3\%) admitted being aware of their hospital's ICU preventive equipment maintenance program (Table 2). Approximately $95.5 \%$ of the nurses believed that the biomedical engineering unit was responsible for PPM in their units, and only $1.4 \%$ believed it was the responsibility of other staff members (physicians and vendors). When asked to rate their comfort level on a Likert-type rating scale, the nurses had a high selfreported comfort level in dealing with equipment in their ICU (mean comfort level $>4 / 5$ ). As shown in Table 2, most nurses $(96.2 \%)$ had received training on how to use infusion pumps, followed by cardiac monitoring systems (78.0\%), and then cardiac defibrillation devices (73.9\%).

\section{Super Users}

Of the 297 respondents, $60(20.2 \%)$ indicated that at least one super user was available for at least one device (Table 2). Fifty-five percent of the respondents indicated the
Table I Respondents' Demographic and Professional Characteristics $(\mathrm{N}=297)$

\begin{tabular}{|l|l|l|}
\hline Variables & Frequency & Percentage \\
\hline Gender & 277 & \\
Female & 20 & 93.3 \\
Male & & 6.7 \\
\hline Experience, years & $7 I$ & \\
I-2 years & 74 & 23.9 \\
3-5 years & 78 & 24.9 \\
6-I0 years & 74 & 26.3 \\
$>$ I0 years & & 24.9 \\
\hline Clinical role & 294 & \\
Nurse & 3 & 99.0 \\
Head nurse & & 1.0 \\
\hline Discipline/working unit & 87 & \\
Medical ICU & 65 & 29.3 \\
Surgical ICU & 87 & 21.8 \\
Pediatric ICU & 58 & 29.3 \\
Neonatal ICU & & 19.5 \\
\hline
\end{tabular}

Abbreviation: ICU, intensive care unit.

existence of super users for infusion pumps, $63.3 \%$ for mechanical ventilators, $53.3 \%$ for cardiac monitors, $51.7 \%$ for electrocardiogram devices, and $48.3 \%$ for cardiac defibrillators.

\section{User Manual}

Overall, 160 out of the 297 nurses (53.9\%) selected one device whose user manual was available. In most cases $(89.6 \%)$, nurses indicated the availability of a user manual for infusion pumps, followed by that for mechanical ventilators $(86.0 \%)$ and cardiac monitors (81.1\%).

\section{Source of Updates}

The nurses' responses varied regarding the source of updates for the indicated devices in Table 3 (infusion pump, mechanical ventilator, cardiac monitor, electrocardiogram, and defibrillator). In most cases, the nurses reported having no resource for updates about the medical devices in their units.

\section{Roles and Responsibilities}

Regarding the management and maintenance of medical equipment, more than half of the respondents $(52.1 \%)$ indicated that biomedical engineers were responsible for assembling the equipment $(\mathrm{p}<0.001)$. Most respondents $(66 \%)$ also reported that biomedical engineers were 
Table 2 Nurses' Perceptions of Periodic Preventive Maintenance and Equipment Training Characteristics and Factors

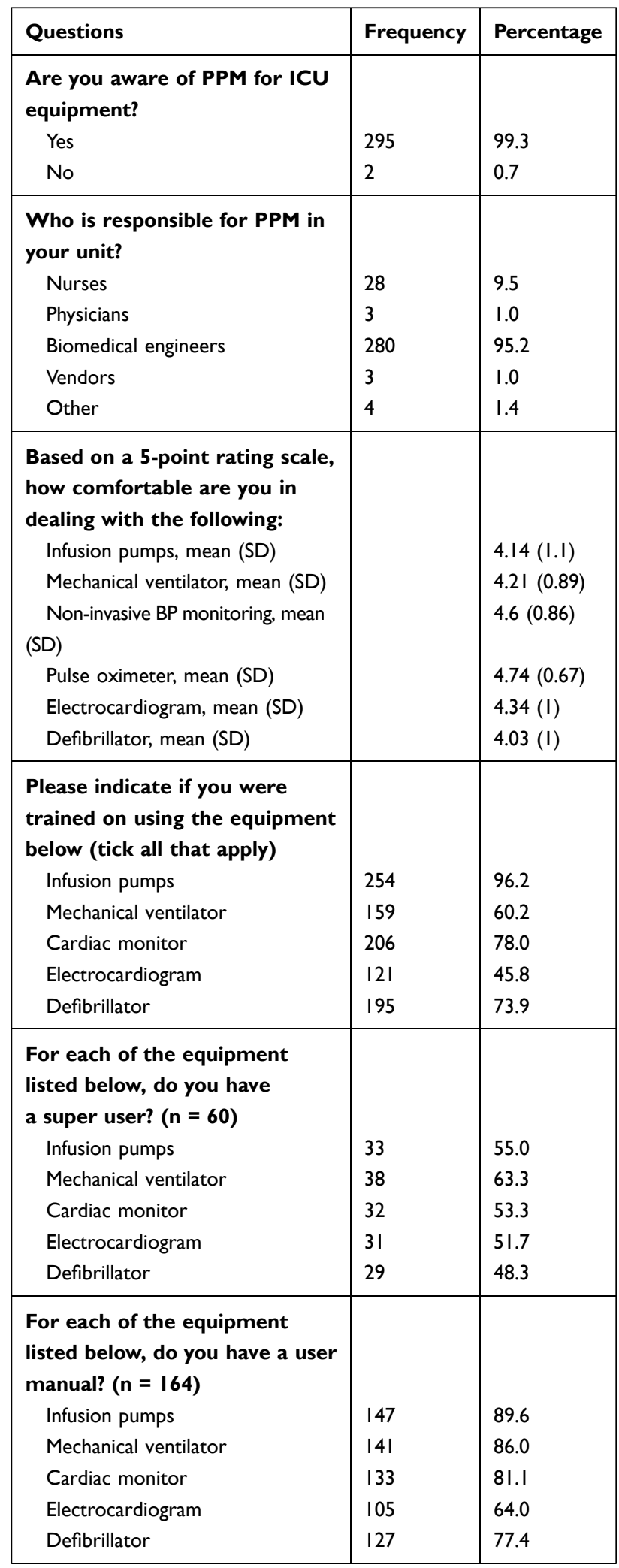

Abbreviations: BP, blood pressure; ICU, intensive care unit; PPM, periodic preventive maintenance; SD, standard deviation. responsible for equipment troubleshooting ( $p<0.001$ ). Regarding the ordering of medical supplies, $64.5 \%$ of the nurses responded that it was the head nurse's responsibility. Conversely, most nurses $(86.3 \%)$ reported that biomedical engineers were responsible for maintaining and calibrating medical devices $(\mathrm{p}<0.001)$

A significant proportion of nurses (78.4\%) cited bedside nurses as those responsible for sterilizing and processing recyclable equipment $(\mathrm{p}<0.001)$. In contrast, most nurses $(58 \%)$ responded that biomedical engineers were responsible for the disposal of non-recyclable items $(p<0.001)$ (Table 4).

\section{Discussion}

The technical relationship between users, medical devices, and biomedical engineering staff in Saudi Arabia has been standardized and considered part of the accreditation requirements for healthcare institutions. These accreditation requirements were issued under the umbrella of the Saudi Arabia Central Board for Accreditation of Healthcare Institutions (CBAHI), ${ }^{3}$ which also ensures that healthcare institutions comply with their requirements.

\section{Standards for Safe Operation}

Although CBAHI has certain requirements for the safety of medical devices, these not considered an Essential Safety Requirement. ${ }^{7}$ In contrast to other international regulations, such as those in the US and Germany, these requirements are obligatory, regardless of the overall weighted scoring criteria. $^{7}$

One aspect addressed in the CBAHI standard is ensuring that hospitals have policies and procedures that regulate the management of medical devices. However, the role and responsibilities assigned to each member of the clinical and technical staff are not well explained. Nevertheless, the standard necessitates in that newcomers should attend an orientation program before working independently (section HR.7.1.3) ${ }^{3}$ and states that the content of the orientation program should include the safe operation and troubleshooting of medical devices.

Another factor that contributes massively to the safe operation of medical devices is the reporting of adverse medical device events. According to the CBAHI standards, HCWs should be aware of the official national requirements and channels for reporting adverse events. Here in Saudi Arabia, the national reporting system for adverse events related to medical devices is a web-based 
Table 3 Nurses' Resources for Further Information on Medical Equipment

\begin{tabular}{|l|l|l|l|l|l|}
\hline Variables & No Resource & Head Nurse & Senior Nurses & Biomedical Engineer & Vendor \\
\hline Infusion pump & $246(82.8 \%)$ & $3(1.0 \%)$ & $12(4.0 \%)$ & $3(1.0 \%)$ & $33(11.0 \%)$ \\
\hline Mechanical ventilator & $249(83.8 \%)$ & $4(1.3 \%)$ & $17(5.7 \%)$ & $0(0.0 \%)$ & $27(9.1 \%)$ \\
\hline Cardiac monitor & $255(85.9 \%)$ & $1(0.3 \%)$ & $21(7.1 \%)$ & $0(0.0 \%)$ & $20(6.7 \%)$ \\
\hline Electrocardiogram & $262(88.2 \%)$ & $1(0.3 \%)$ & $15(5.1 \%)$ & $0(0.0 \%)$ & $19(6.4 \%)$ \\
\hline Defibrillator & $260(87.5 \%)$ & $0(0.0 \%)$ & $13(4.4 \%)$ & $1(0.3 \%)$ & $23(7.7 \%)$ \\
\hline
\end{tabular}

Table 4 Nurses' Perceived Responsibilities for Various Medical Equipment Maintenance Processes and Transactions

\begin{tabular}{|l|l|l|l|l|l|l|}
\hline Variables & Nurses & Head Nurses & Physicians & Biomedical Engineers & Manufacturers & p-value \\
\hline Equipment assembly & $66(23.2 \%)$ & $13(4.6 \%)$ & $7(2.5 \%)$ & $148(52.1 \%)$ & $50(16.6 \%)$ & $<0.00 \mathrm{I}$ \\
\hline Troubleshooting equipment & $76(26.6 \%)$ & $\mathrm{I}(0.3 \%)$ & $7(2.4 \%)$ & $190(66.0 \%)$ & $3(\mathrm{I} .0 \%)$ & $<0.00 \mathrm{I}$ \\
\hline Ordering supplies & $28(9.4 \%)$ & $182(64.5 \%)$ & $\mathrm{II}(3.5 \%)$ & $50(17.7 \%)$ & $7(2.5 \%)$ & $<0.00 \mathrm{I}$ \\
\hline Maintenance and device calibration & $24(8.4 \%)$ & $4(1.4 \%)$ & $4(1.4 \%)$ & $246(86.3 \%)$ & $4(\mathrm{I} .4 \%)$ & $<0.00 \mathrm{I}$ \\
\hline Sterilization of recyclables & $2 \mathrm{II}(78.4 \%)$ & $4(\mathrm{I} .4 \%)$ & $\mathrm{I}(0.4 \%)$ & $44(16.4 \%)$ & $7.6 \%)$ & $<0.00 \mathrm{I}$ \\
\hline Disposal of non-recyclable items & $74(26.3 \%)$ & $31(\mathrm{II} .0 \%)$ & $0(0.0 \%)$ & $163(58 \%)$ & $13(4.6 \%)$ & $<0.00 \mathrm{I}$ \\
\hline
\end{tabular}

electronic system governed by the Saudi Food and Drug Authority (SFDA). ${ }^{8-10}$

\section{PPM and Procedures Related to Medical Devices}

The responsibilities for the different tasks related to medical devices and equipment, such as PPM, sterilization, disposal of non-recyclable items, and ordering supplies, were well-recognized and coordinated between the different team members from the ICUs and the biomedical engineering department, as indicated in the respondents' answers. Notably, most of the respondents were aware of their roles and that of the biomedical engineering department. Such communication and multidisciplinary teamwork are important to optimize the management of medical devices in ICUs. ${ }^{11}$

It would be helpful to create a computer-based inventory system of all ICU devices accessible to all departments involved in the safe handling, processing, and managing of medical devices with in healthcare facilities. Such a system would lead the multidisciplinary effort to maintain the safety and traceability of medical devices from all aspects.
The system can include educating users on the utilization and provision of checklists. Additionally, it can help the staff determine when PPM is required and enhance the technical follow-up of devices, maintenance and repair logs, and the ability to promptly provide users with replacement devices. Lastly, there should be criteria decide when a device should be discarded.

\section{Existence of Super Users}

The HCWs indicated that when they want to learn more about a medical device within their unit, they tend to contact the device manufacturer first, followed by the designated super users. The majority indicated that their units had no device super users, and when they are occasionally present, their specific role as a super user is not defined. This finding highlights the need for the official assignment of a super user for each device type or model in every unit. The super user could solve issues faced by the medical staff by leading all aspects related to the use and operation of the device as well as mentoring and training other users in-house.

The role of the super user, as identified in the German Medical Devices Operation Ordinance Medical Devices Clinical Educator, ${ }^{7}$ is crucial for different aspects related 
to the safe operation of the medical devices assigned to the super-user. These obligations include attending training sessions on the safe operation of these medical devices that qualify the super user to train other users within the healthcare institution, organizing training on the use of the medical devices for other HCWs, documenting all the activities related to the medical devices, assuring the availability of the instructions for use of the medical devices, and monitoring PPM compliance.

Other roles for the super user might include organizing discussions to address safety issues regarding the operation of these medical devices with the users, competent authority officers (such as the SFDA), the manufacturer, the biomedical engineering department, and other departments involved in the operation of these medical devices (such infection control). Super users should also support the competent authority officer in preparing procedures for reporting adverse events, applying recall procedures for medical devices, and facilitating investigations.

\section{Responsibilities and Role of Technical Staff and Troubleshooting}

Any technical error encountered during the use of a medical device could have a highly negative impact on the safety of the patient or user. Our survey found that $8 \%$ of respondents tended to immediately reboot the system once the alarm went off as the first step, $32 \%$ considered rebooting the device as a second step, and $22 \%$ considered it as the third step. A survey among ICU nurses in the USA showed that up to $40 \%$ of nurses had never heard of or used 27 functions on the monitors. ${ }^{8}$ Ideally, when the alarm on a medical device goes off, the typical action is for users to respond and manage it according to the procedure standards that they learned during training for that specific medical device. If they fail to identify and resolve the issue, they should consult the assigned super user. If the issue persists, they should contact the biomedical engineering department and then the manufacturer to manage it properly. It is risky to attempt to reboot and reuse the device when it starts functioning again because rebooting could keep failures hidden, which could result in a serious adverse event if the issue is not identified and managed properly. Periodic, personalized training is essential for safer use of ICU monitors and to reduce alarm fatigue. ${ }^{8}$ A multi-faceted involvement of clinicians, industry, computer scientists, and regulatory agencies was advocated to prevent alarm fatigue among HCWs. ${ }^{9}$

\section{Risk Information Communication}

Most of our respondents relied heavily on the manufacturer's feedback for updates on the risks related to medical devices. A small proportion relied on their colleagues and the medical literature to receive updates on the risks related to these devices. Of note, only six respondents (2\%) considered the SFDA to be a source of information about the risk of medical devices. This low percentage could be due to a lack of awareness about the role of the SFDA as a source of medical device risk information and recalls. ${ }^{2}$ The apparent lack of knowledge among the respondents could also be due to a lack of communication between the SFDA and healthcare providers.

\section{Conclusion}

This study showed awareness of ICU HCWs of the importance of the effective operation of the medical equipment, but they lack some knowledge of the safe operation of medical devices in regard to the technical aspects. Updates regarding the risk information of ICU medical devices and supporting super users is advised. This study also highlights the need for a framework for safer operation of medical devices that includes empowerment of super users and ICU HCWs, and optimizing the communication between the national regulatory bodies of medical devices and the healthcare providers. Replicating this study in other healthcare settings is suggested as another quality improvement project.

\section{Abbreviations}

BP, blood pressure; CBAHI, Central Board for Accreditation of Healthcare Institutes; HCW, healthcare worker; ICU, intensive care unit; KSUMC, King Saud University Medical City; PPM, periodic preventive maintenance; SD, standard deviation; SFDA, Saudi Food and Drug Authority.

\section{Data Sharing Statement}

All data generated or analyzed during this study are included in this published article.

\section{Ethical Approval and Consent to Participate}

Written informed consent was obtained from all participants. Participation was voluntary, and all participants were assured that their confidentiality would be protected. The Institutional Review Board of King Saud University granted permission to conduct this survey. 


\section{Acknowledgments}

We thank all the ICU nurses working at King Saud University Medical City who participated in this study. Fahad Alsohime's current address is College of Medicine, King Saud University, Riyadh, Saudi Arabia. Omar Alsohime's current address is Standards and Guidelines Department, Medical Devices Sector, Saudi Food and Drug Authority, Riyadh, Saudi Arabia.

\section{Author Contributions}

All authors made a significant contribution to the work reported, whether that is in the conception, study design, execution, acquisition of data, analysis and interpretation, or in all these areas; took part in drafting, revising or critically reviewing the article; gave final approval of the version to be published; have agreed on the journal to which the article has been submitted; and agree to be accountable for all aspects of the work.

\section{Funding}

This work was supported by the Deanship of Scientific Research, King Saud University, Riyadh, Saudi Arabia. This research has been financially supported by Prince Abdullah Ben Khalid Celiac Disease Research Chair, under the Vice Deanship of Research Chairs, King Saud University, Riyadh, Kingdom of Saudi Arabia. The funder had no role in the study design, data collection and analysis, decision to publish, or preparation of the manuscript.

\section{Disclosure}

The authors report no conflicts of interest in this work.

\section{References}

1. Frey B, Doell C, Klauwer D, et al. The morbidity and mortality conference in pediatric intensive care as a means for improving patient safety. Pediatr Crit Care Med. 2016;17:67-72. doi:10.1097/ PCC.0000000000000550

2. Alsohime F, Temsah M-H, Hasan G, et al. Reporting adverse events related to medical devices: a single center experience from a tertiary academic hospital. PLoS One. 2019;14(10):e224233. doi:10.1371/ journal.pone. 0224233

3. The Saudi Central Board for Accreditation of Healthcare Institutions (CBAHI). CBAHI-national hospital standards: 3rd ed 1436-2015. Saudi Central Board for Accreditation of Healthcare Institutions; 2015.

4. Cuthrell P. Managing equipment failures: nursing practice requirements for meeting the challenges of the Safe Medical Devices Act. J Intraven Nurs. 1996;19:264-268.

5. Bourgain JL, Coisel Y, Kern D, Nouette-Gaulain K, Panczer M. ventilator group of the French Society of Anaesthesia, Intensive Care. What are the main "machine dysfunctions" to know? Ann Fr Anesth Reanim. 2014;33:466-471. doi:10.1016/j.annfar.2014.07.744

6. Zippel C, Börgers A, Weitzel A, Bohnet-Joschko S. Many critical incidents could be avoided by preanaesthesia equipment checks: lessons for high reliability organisations. Eur $J$ Anaesthesiol. 2014;31(5):289-291. doi:10.1097/EJA.0000000000000054

7. The German ordinance for operating medical devices in healthcare from the Federal Institute for drugs and medical devices (bundesinstitut für arzneimittel und medizinprodukte, bfarm) (aami Q56). [Internet]. 1998 [cited April 14, 2020]. Available from: http://www. gesetze-im-internet.de/mpbetreibv/index.html. Accessed April 2, 2021.

8. Sowan AK, Vera AG, Fonseca EI, Reed CC, Tarriela AF, Berndt AE. Nurse competence on physiologic monitors use: toward eliminating alarm fatigue in intensive care units. Open Med Inform J. 2017;11:1-11. doi:10.2174/1874431101711010001

9. Hravnak M, Pellathy T, Chen L, et al. A call to alarms: current state and future directions in the battle against alarm fatigue. J Electrocardiol. 2018;51(6S):S44-S48. doi:10.1016/j.jelectrocard.2018.07.024

10. Sezdi M. Performance Analysis for Medical Devices. Biomedical Engineering Research. 2013;2(3):139-146. doi:10.5963/ BER0203003

11. Sezdi M. Medical Technology Management and Patient Safety. In: $A$ Roadmap of Biomedical Engineers and Milestones. First Edition. Intech. 2012:183-208. doi:10.5772/35926

\section{Publish your work in this journal}

The Journal of Multidisciplinary Healthcare is an international, peerreviewed open-access journal that aims to represent and publish research in healthcare areas delivered by practitioners of different disciplines. This includes studies and reviews conducted by multidisciplinary teams as well as research which evaluates the results or conduct of such teams or healthcare processes in general. The journal covers a very wide range of areas and welcomes submissions from practitioners at all levels, from all over the world. The manuscript management system is completely online and includes a very quick and fair peer-review system. Visit http://www.dovepress.com/testimonials. php to read real quotes from published authors. 